ISSN 0258-7122

Bangladesh J. Agril. Res. 36(3) : 415-426, September 2011

\title{
EFFECT OF HIGH TEMPERATURE ON YIELD ATTRIBUTING TRAITS IN BREAD WHEAT
}

\author{
KHAJAN SINGH $^{1}$, S. N. SHARMA ${ }^{1}$ AND YOGENDRA SHARMA ${ }^{2}$
}

\begin{abstract}
High temperature stress is major constraint to bread wheat (Triticum aestivum L. Em. Thell) production. Generation of information on the effect of high temperature stress on various traits may be helpful for developing thermotolerance bread wheat variety. An experiment was conducted on a set of 10 diverse genotypes, their $45 \mathrm{~F}_{1} \mathrm{~S}$ and $\mathrm{F}_{2} \mathrm{~S}$ for identification of high temperature stress genotype. The experiment was conducted under normal and late sown condition. The parent HD 2851, P8W 520, and HS 448, and the crosses HS 448 $\times$ PBW 520, UP $2614 \times$ K 209 and PBW $520 \times$ HD 2851 for grain yield per plant were least affected under late sown conditions. Heat stress intensity (Dvalue) clearly indicated that grain yield per pant biological yield per plant and grain yield per spike suffered revively under late sown conditions.
\end{abstract}

Keywords: Bread wheat, heat susceptibility index, tolerant genotypes.

\section{Introduction}

Wheat is a self-pollinated crop originated in South East Asia. It is the second main source of world's food energy and nutrition. It is grown on about 221.70 million hectares in a range of environments, with annual production of about 655.8 million tonnes (FAO, 2010). India is the second largest wheat producing nation and contributes approximately $11.83 \%$ (77.60 million tonnes) to the world wheat production from about $12.50 \%$ (27.82 million hectares) of global area during 2009-2010 (FAO, 2010). The major wheat producing countries are China, India, USA, the Russian Federation, and Australia. These five countries together contribute more than half of the global wheat production.

During the last four decades of the $20^{\text {th }}$ century, the global wheat production is doubled from 3 to 6 billion and it is estimated that by the year 2012, it will reach 8 billion (Prasad and Nagarajan, 2004). Globally, demand for wheat by the year 2020 is forecasted around 950 million tonnes to meet future demands imposed by population growth. This target will be achieved only, if global wheat production is increased by $2.5 \%$ per annum. This must he achieved under reduced water availability, a scenario of global warming, and evolving pathogen and pest populations.

\footnotetext{
${ }^{1}$ All India Coordinated Wheat and Barley Improvement Project, S. K. Rajasthan Agriculture University, Agricultural Research Station, Durgapura, Jaipur 302018. Rajasthan, India, ${ }^{2}$ Energy Seed International Pvt. Ltd., 309, Shekhawati Complex, Station Road, Jaipur - 302 006, Rajasthan, India.
} 
Breeding wheat cultivars with increased grain yield potential, enhanced water use efficiency, heat tolerance, end use quality, and durable resistance to important diseases and pests can contribute to meeting at least half of the desired production increases. The remaining half must come through better agronomic and soil management practices and incentive policies.

Yield potential of any variety is the combined effect of genotypes and environmental interaction. Now a days, due to global warming, climatic conditions are changing and temperature begins to rise in late February and March coupled with hot dry winds during the post anthesis stages, especially during grain development terminate grain growth prematurely and reduces yield considerably. Hence, there is need to bred new genotypes having genetic mechanism to tolerate high temperature by involving novel genes from diverse parents to sustain and maximize productivity of wheat under warmer areas of India.

With the view of the major constraints lying to achieve higher yield plateau in wheat, there is further need to develop high yielding and rust resistant wheat varieties suitable for mega environments of the country. To enhance the production and productivity in wheat cultivation particularly in warmer areas, new set of varieties having heat tolerance are required. Breeding of heat stress tolerance forms is an integral component of wheat breeding programmes at both national and international level. Thermo-tolerance in wheat cultivars requires understanding of the physiological responses of wheat crop of this stress, which will help in identifying traits, to be used as selection criteria. The present study was carried out to understand the effect on yield attributing traits under high temperature conditions and identification of tolerant genotypes suitable for this environment that the yield target can be met out under changing conditions.

\section{Materials and Method}

Ten diverse genotypes of bread wheat (Triticum aestivum L. em. Thell.), namely, HD 2881, HS 448, WI-I 789, HUW 468, Up 2614, NW 3015, PBW 520, K 209, HD 2851, and Raj 4063 were selected on the basis of a broad range of genetic diversity for major yield components, geographical origin and their suitability for different yield traits. The experiment was conducted during the rabi season at Research Farm of Agricultural Research Station, Durgapura, Jaipur. It is situated at $26^{\circ} 51^{\prime}$ ' N latitude and $75^{\circ} 47^{\prime} \mathrm{E}$ longitude and an altitude of 300 meters above mean sea level in Rajasthan, India.

Ten parents and their resulting $45 \mathrm{~F}_{1} \mathrm{~S}$ and $45 \mathrm{~F}_{2} \mathrm{~S}$ were grown in a randomized block design with three replications under normal $\left(\mathrm{E}_{1}-20^{\text {th }}\right.$ November), and very late $\left(\mathrm{E}_{2}-20^{\text {th }}\right.$ December $)$ sown conditions. In each replication, parents and $F_{1}$ s were sown by dibbling the seed in a plot of two rows of $4 \mathrm{~m}$ length and the $\mathrm{F}_{2} \mathrm{~S}$ in a plot of six rows of $3 \mathrm{~m}$ length in each environment. 
A spacing of $30 \mathrm{~cm} \times 10 \mathrm{~cm}$ was maintained in all the plots for row to row and plant to plant. Non-experimental rows were planted all around the experimental plot to avoid border effects. Recommended plant protection procedures were followed for raising the crop in all the environments. Observations were recorded on 20 randomly selected plants from each replication in case of parents and $F_{1} s$, while sixty in $F_{2}$ generation in both environments separately for 14 morphological/agronomical parameters viz., days to heading, days to maturity, plant height $(\mathrm{cm})$, number of tillers per plant, flag leaf area $\left(\mathrm{cm}^{2}\right)$, peduncle length $(\mathrm{cm})$, spike length $(\mathrm{cm})$, number of spikelets per spike, number of grains per spike, grain yield per spike (g), 1000-grain weight (g), biological yield per plant (g), harvest index (\%), and grain yield per plant (g).

Heat susceptibility index (HSI) was calculated for grain yield and other attributes over high temperature stress (late sown) and non-stress environment (normal sown) by using the formula as suggested by Fischer and Maurer (1978).

$\mathrm{HSI}=[\mathrm{l}-\mathrm{YD} / \mathrm{YP}] / \mathrm{D}$

Where,

$\mathrm{YD}=$ mean of the genotypes in stress environment.

$\mathrm{YP}=$ mean of the genotypes under non-stress environment.

$\mathrm{D}=1$-[mean YD of all genotypes/mean YP of all genotypes].

The HSI values were used to characterize the relative tolerance of genotypes based on minimization of yield losses compared to normal environmental conditions. The differences between genotypes for different characters were tested for significance by using standard techniques for analysis of variances.

\section{Results and Discussion}

The results obtained from the analysis of variance studies showed highly significant differences among all the characters and genotypes in all the sowing environments indicating the influence of sowing condition on genotypes and traits. Further, it was observed that all the characters respond to high temperature stress in different way in different genotypes.

The mean of parents, $F_{1} s$ and $F_{2} s$ for different characters decreased tinder $F_{2}$ environment (late sown) in comparison to $\mathrm{E}_{1}$ environment (normal sown) [Table 1]. The results are in agreement with Nagarajan and Rane (2002), Rashid et al. (2004), Vivek Raj and Bhardwaj (2004) and Ved Prakash (2007). Perusal of Table 2 indicated that parent, WH 789, HS 448, HUW 468, and Raj 4063 for days to heading; HS 448, NW 3015, UP 2614, HUW 468, and WH 789 tbr days to maturity; HS 448, Raj 4063, WH 789, and PBW 520 for plant height; HS 448, PBW 520, UP 2614, and K 209 for effective tillers/plant; Raj 4063, HD 
2881, HS 448, and PBW 520 for flag leaf area; Raj 4063, UP 2614, and PBW 520 for peduncle length; HUW 468, PBW 520, HD 2881, and Raj 4063 for spike length; NW 3015, HD 2881, and UP 2614 for number of spikelets/spike; HD 2851, NW 3015, and HUW 468 for number of grains/spike; HD 2851 and PBW 520 for grain yield/spike; WH 789, PBW 520, HD 2881, HS 448, and HD 2851 for 1000-grain weight; HD 2851, UP 2614 and PBW 520 for biological yield/ plant; PBW 520, HS 448, and HD 2881 for harvest index and HD 2851, PBW 520 and HS 448 for grain yield/plant were least affected under late sown conditions $\left(E_{2}\right)$. An overall appraisal indicated that HD 2851, PBW 520, and HS 448 were found to be good parents for grain yield per plant based on HSI.

Table 1. Mean of parents, their $F_{1}$ sand $F_{2} s$ for various characters in normal $\left(E_{1}\right)$ and late sown condition $\left(E_{2}\right)$ in bread wheat.

\begin{tabular}{|c|c|c|c|c|}
\hline Characters & Environments & Parents & $\mathrm{F}_{1}$ crosses & $\mathrm{F}_{2}$ crosses \\
\hline \multirow[t]{2}{*}{ Days to heading } & $\mathrm{E}_{1}$ & 84.70 & 83.89 & 84.67 \\
\hline & $\mathrm{E}_{2}$ & 78.77 & 79.92 & 75.59 \\
\hline \multirow[t]{2}{*}{ Days to maturity } & $\mathrm{E}_{1}$ & 119.50 & 119.10 & 119.21 \\
\hline & $\mathrm{E}_{2}$ & 107.87 & 107.60 & 107.49 \\
\hline \multirow[t]{2}{*}{ Plant height (cm) } & $\mathrm{E}_{1}$ & 78.13 & 79.56 & 78.86 \\
\hline & $\mathrm{E}_{2}$ & 74.60 & 71.65 & 70.10 \\
\hline \multirow[t]{2}{*}{ Tillers/plant } & $\mathrm{E}_{1}$ & 4.81 & 5.29 & 5.11 \\
\hline & $\mathrm{E}_{2}$ & 4.26 & 4.43 & 4.29 \\
\hline \multirow[t]{2}{*}{ Flag leaf area $\left(\mathrm{cm}^{2}\right)$} & $\mathrm{E}_{1}$ & 22.56 & 26.46 & 24.93 \\
\hline & $\mathrm{E}_{2}$ & 17.25 & 16.94 & 16.73 \\
\hline \multirow[t]{2}{*}{ Peduncle length (cm) } & $\mathrm{E}_{1}$ & 27.33 & 30.23 & 30.49 \\
\hline & $\mathrm{E}_{2}$ & 23.31 & 21.87 & 21.69 \\
\hline \multirow[t]{2}{*}{ Spike length (cm) } & $\mathrm{E}_{1}$ & 10.56 & 12.25 & 11.74 \\
\hline & $\mathrm{F}_{2}$ & 9.78 & 10.89 & 10.71 \\
\hline \multirow[t]{2}{*}{ No. of spikelets/spike } & $\mathrm{E}_{1}$ & 17.21 & 19.24 & 18.91 \\
\hline & $\mathrm{E}_{2}$ & 16.12 & 18.02 & 17.89 \\
\hline \multirow[t]{2}{*}{ No. of grains/spike } & $\mathrm{E}_{1}$ & 47.58 & 54.15 & 51.81 \\
\hline & $\mathrm{F}_{2}$ & 40.62 & 45.89 & 44.50 \\
\hline \multirow[t]{2}{*}{ Grain yield/spike (g) } & $\mathrm{E}_{1}$ & 1.80 & 2.16 & 1.95 \\
\hline & $\mathrm{E}_{2}$ & 1.26 & 1.53 & 1.39 \\
\hline \multirow[t]{2}{*}{ 1000- grain weight (g) } & $\mathrm{E}_{1}$ & 38.02 & 40.07 & 38.07 \\
\hline & $\mathrm{E}_{2}$ & 31.41 & 33.70 & 31.38 \\
\hline \multirow[t]{2}{*}{ Biological yield/plant (g) } & $\mathrm{E}_{1}$ & 22.25 & 28.64 & 26.21 \\
\hline & $\mathrm{E}_{2}$ & 15.70 & 18.04 & 16.09 \\
\hline \multirow[t]{2}{*}{ Harvest index (\%) } & $\mathrm{E}_{1}$ & 34.20 & 36.23 & 35.08 \\
\hline & $\mathrm{E}_{2}$ & 30.29 & 34.68 & 33.49 \\
\hline \multirow[t]{2}{*}{ Grain yield/plant (g) } & $\mathrm{E}_{1}$ & 7.61 & 10.35 & 9.19 \\
\hline & $\mathrm{E}_{2}$ & 4.77 & 6.25 & 5.32 \\
\hline
\end{tabular}


Among the $\mathrm{F}_{1}$ crosses, HD $2881 \times$ WH 789, HD $2881 \times$ PBW 520, and HD $2881 \times$ HD 2851 for days to heading; HS $448 \times$ Up 2614, HS $448 \times$ HUW 468, HS $448 \times$ NW 3015, and HS $448 \times$ PBW 520 for days to maturity; HD $2851 \times$ Raj 4063, WH $789 \times$ HUW 468, HUW $468 \times$ Raj 4063, HUW $468 \times$ HD 2851, and PBW $520 \times$ Raj 4063 for plant height; WH $789 \times$ PBW 520, HD $2881 \times$ HS 448, HUW $468 \times$ K 209, HS $448 \times$ NW 3015, and HUW $468 \times$ PBW 520 for effective tillers/plant; PBW $520 \times$ HD 2851, HD $2851 \times$ Raj 4063; HS $448 \times$ NW 3015, and HUW $468 \times$ RD 2851 for flag leaf area; UP $2614 \times$ PBW 520, Up $2614 \times$ Raj 4063, HD $2881 \times$ Raj 4063, and HS $448 \times$ HD 2851 for peduncle length; UP $2614 \times$ Raj 4063, HS $448 \times$ NW 3015, UP $2614 \times$ PBW 520, HS 448 $\times$ PBW 520, WR $789 \times$ HD 2851, and UP $2614 \times$ PBW 520 for spike length; UP $2614 \times$ Raj 4063, HS $448 \times$ WH 789, HS $448 \times$ NW 3015, HD $2881 \times$ NW 3015, HS $448 \times$ HUW 468, and WH $789 \times$ HD 2851 for number of spikelets/spike; HUW $468 \times$ Raj 4063, HD $2881 \times$ UP 2614, HUW $468 \times$ HD 2851, WH $789 \times$ HUW 468, and HUW $468 \times$ HD 2851 for number of grains/spike; HUW $468 \times$ HD 2851, PBW $520 \times$ HD 2851, and UP 2614 x K 209 for grain yield/spike; UP $2614 \times$ PBW 520, HUW $468 \times$ Raj 4063, HUW $468 \times$ HD 2851 and K $209 \times$ Raj 4063 for 1000-grain weight; HUW $468 \times$ HD 2851, HS $448 \times$ PBW 520, WH $789 \times$ PBW 520, HUW $468 \times$ K 209, and NW $3015 \times$ P8W 720 for biological yield/plant; PBW $520 \times$ K 209, UP $2614 \times$ HD 2851, UP $2614 \times$ K 209, and HD $2881 \times$ PBW 520 for harvest index and HS $448 \times$ PBW 520, UP $2614 \times$ K 209, HUW $468 \times$ HD 2851, and PBW $520 \times$ HD 2851 for grain yield/plant were least affected under late sown conditions $\left(\mathrm{E}_{2}\right)$. In general, HS $448 \times$ PBW 520, UP $2614 \times \mathrm{K} 209$ and HUW $468 \times$ HD 2851 were found to be good $\mathrm{F}_{1}$ crosses for grain yield/plant based on HSI (Table 3 ).

The crosses K $209 \times$ Raj 4063, HD $2881 \times$ PBW 520 and HD $2881 \times$ HD 2851 for days to heading; HS $448 \times$ PBW 520, HUW $468 \times$ HD 2851, HS $448 \times$ K 209, and WH $789 \times$ HD 2851 for days to maturity: HD $2851 \times$ Raj 4063, UP $2614 \times$ Raj 4063, HS $448 \times$ HD 2851 and HS $448 \times$ NW 3015 for plant height; UP $2614 \times$ PBW 520, WH $789 \times$ HUW 468, HS $448 \times$ UP 2614, and HUW 468 $\times$ PBW 520 for effective tillers/plant; PBW $520 \times$ Raj 4063, HS $448 \times$ NW 3015, and WH $789 \times$ HD 2851 for flag leaf area; UP $2614 \times$ PBW 520, HS $448 \times$ HD 2851, WH $789 \times$ K 209, and HD $2881 \times$ Raj 4063 for peduncle length; HS $448 \times$ HUW 468, HUW $468 \times$ Raj 4063, HD $2881 \times$ K 209 and PBW $520 \times$ HD 2851 for spike length; HUW $468 \times$ Raj 4063, UP $2614 \times$ HD 2851, HS $448 \times$ WH 789 and PBW $520 \times$ Raj 4063 for number of spikelets/spike; UP $2614 \times$ NW 3015, HD $2881 \times$ K 209, HD $2881 \times$ UP 2614. HD $2881 \times$ NW 3015, and WH $789 \times$ UP 2614 for number of grains/spike; WH $789 \times$ Raj 4063, HUW $468 \times$ HD 2851, and WH $789 \times$ HD 2851 for grain yield/spike; UP $2614 \times$ PBW 520, HD $2881 \times$ HD 2851, and WH $789 \times$ NW 3015 for 1000-grain weight; UP $2614 \times$ PBW 520, WH $789 \times$ K 209, UP $2614 \times$ NW 3015, HUW $468 \times$ K 209, and NW $3015 \times$ PBW 520 for biological yield/plant; UP $2614 \times$ NW 3015, HS $448 \times$ 
PBW 520, HS $448 \times$ NW 3015, WH $789 \times$ HD 2851, and WH $789 \times$ PBW 520 for harvest index and WH $789 \times$ K 209, UP $2614 \times$ PBW 520, and NW $3015 \times$ PBW 520 for grain yield/plant were least affected under late sown condition $\left(E_{2}\right)$. In general, the crosses WH $789 \times$ K 209, UP $2614 \times$ NW 3015, UP $2614 \times \mathrm{PBW}$ 520, and NW $3015 \times$ PBW 520 were found to he good in $F_{2}$ generation for grain yield, plant based on HSI (Table 4).

In order to determine relative tolerance, the heat susceptibility index was estimated for various characters. Based upon the value and direction of desirability, ranking was done for different genotypes as highly heat tolerant (HSI < 0.50), heat tolerant (HSI: 0.51-0.75), moderately heat tolerant (HSI: 0.76-1.00), and heat susceptible (HSI $>1.00$ ). An overall appraisal revealed the parent HD 2851, PBW 520, and HS 448; the $F_{1}$ crosses HS $448 \times$ PBW 520, UP $2614 \times$ K 209, PBW $520 \times$ RD 2851, HUW $468 \times$ HD 2851, UP $2614 \times$ PBW 520, HS $448 \times$ UP 2614, HS $448 \times$ HD 2851, WH $789 \times$ PBW 520, and PBW $520 \times \mathrm{K} 209$ and the $\mathrm{F}_{2}$ crosses UP $2614 \times \mathrm{NW} 3015$, WH $789 \times \mathrm{K} 209$, NW $3015 \times$ PBW 520, HUW $468 \times$ HD 2851, WH $789 \times$ HUW 468 and HS $448 \times$ NW 3015 for grain yield per plant were least affected under late sown conditions. High grain yield of a genotype under late sown condition indicated the presence of genes for heat tolerance. Comparison across the generations indicated that the cross HUW $468 \times$ HD 2851, WH $789 \times$ K 209, and UP 2614 $\times$ PBW 520 emerged as highly heat tolerant in $F_{1}$ and $F_{2}$ for grain yield per plant. Heat stress intensity (D-value) revealed that harvest index, days to heading, number of spikelets/spike, days to maturity, and plant height were less affected by late sown condition, while grain yield per plant, biological yield/plant, flag leaf area, grain yield/spike and peduncle length were highly suffered under $\mathrm{E}_{2}$ environment.

If we consider D-value i.e. heat stress intensity, it was revealed that days to heading, days to maturity, plant height, number of spikelets/spike, spike length, and harvest index were less affected by late sown condition, while grain yield per plant, biological yield per plant, flag leaf area, grain yield per spike and peduncle length highly suffered under $F_{2}$ environment. This clearly indicated that grain yield depends on biological yield and grain yield/spike. Similar results were also reported by Sharma (1993) and Ved Prakash (2007). Blum et al. (1997) emphasized that selection for high biomass yield should bring about positive improvement in grain yield and 1000-grain weight. Thus, biomass yield could he improved by plant height. In the present investigation, plant height significantly contributed towards biomass because less reduction in plant height $(\mathrm{D}=0.05,0.10$ and 0.11 for parents, $F_{1} S$ and $F_{2} S$, respectively) in $E_{2}$ environment. Thus, selection for biomass yield is one of the most important ways to improve the productivity under late sown conditions. 
Table 2. Heat susceptibility indices of parents for various characters in bread wheat.

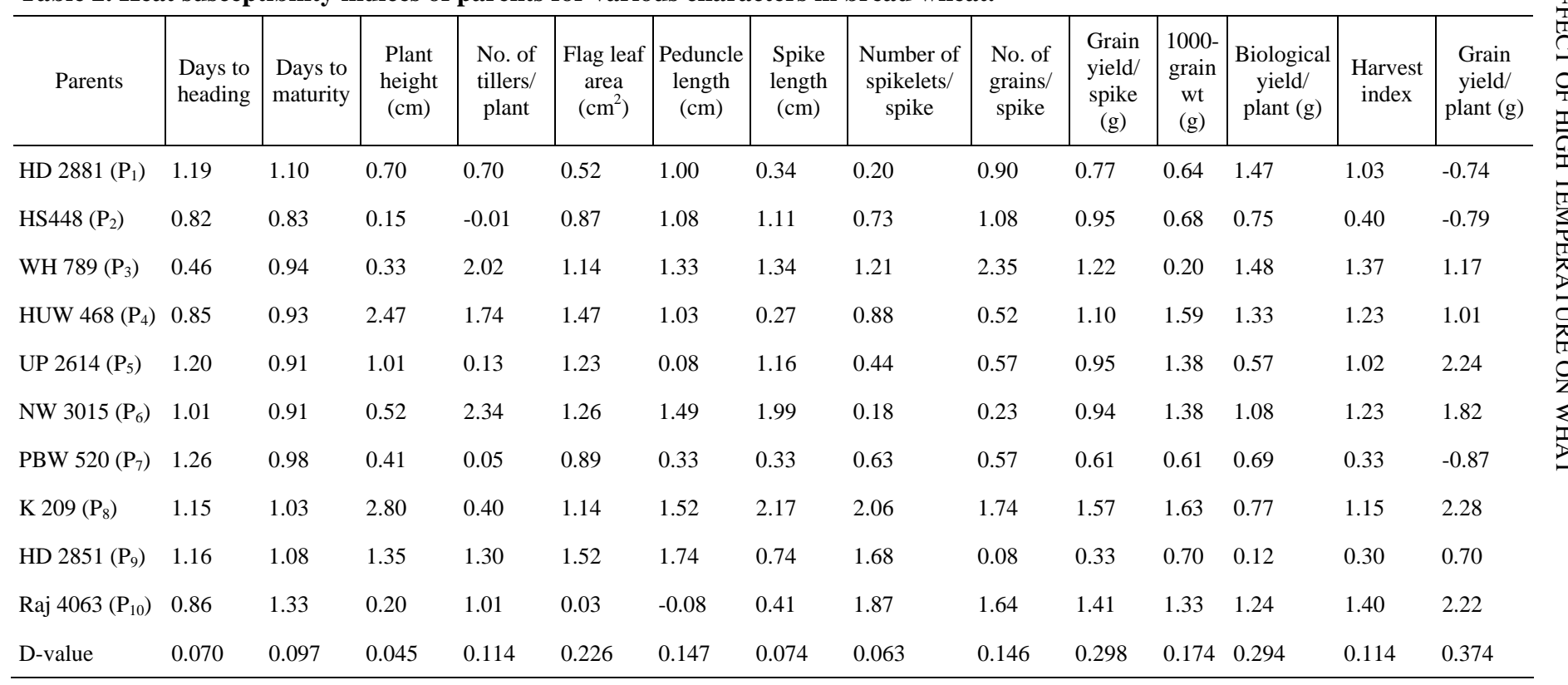

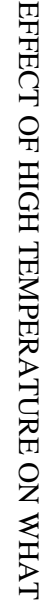


Table 3. Heat susceptibility indices of $F_{1}$ crosses for various characters in bread wheat.

\begin{tabular}{|c|c|c|c|c|c|c|c|c|c|c|c|c|c|c|}
\hline Parents & $\begin{array}{l}\text { Days to } \\
\text { heading }\end{array}$ & $\begin{array}{l}\text { Days to } \\
\text { maturity }\end{array}$ & $\begin{array}{l}\text { Plant } \\
\text { height } \\
(\mathrm{cm})\end{array}$ & $\begin{array}{c}\text { No. of } \\
\text { tillers/ } \\
\text { plant }\end{array}$ & $\begin{array}{c}\text { Flag } \\
\text { leaf } \\
\text { area } \\
\left(\mathrm{cm}^{2}\right)\end{array}$ & $\begin{array}{l}\text { Peduncle } \\
\text { length } \\
\text { (cm) }\end{array}$ & $\begin{array}{l}\text { Spike } \\
\text { length } \\
(\mathrm{cm})\end{array}$ & $\begin{array}{l}\text { No. of } \\
\text { spikelets/ } \\
\text { spike }\end{array}$ & $\begin{array}{l}\text { No. of } \\
\text { grains/ } \\
\text { spike }\end{array}$ & $\begin{array}{c}\text { Grain } \\
\text { yield/ } \\
\text { spike } \\
\text { (g) }\end{array}$ & $\begin{array}{c}1000- \\
\text { grain } \\
\text { wt } \\
(\mathrm{g})\end{array}$ & $\begin{array}{l}\text { Biological } \\
\text { yield / } \\
\text { plant(g) }\end{array}$ & $\begin{array}{c}\text { Harvest } \\
\text { index }\end{array}$ & $\begin{array}{c}\text { Grain } \\
\text { yield/ } \\
\text { plant } \\
\text { (g) }\end{array}$ \\
\hline $\mathrm{P}_{1} \times \mathrm{P}_{2}$ & 0.51 & 1.04 & 1.45 & -0.19 & 1.01 & 1.03 & 0.13 & 0.04 & 1.42 & 1.19 & 1.01 & 0.32 & 0.58 & 2.91 \\
\hline $\mathrm{P}_{1} \times \mathrm{P}_{3}$ & 0.26 & 1.12 & 1.07 & 1.53 & 1.61 & 1.71 & 0.75 & 1.38 & 0.86 & 0.78 & 0.70 & 0.89 & 0.74 & -1.32 \\
\hline $\mathrm{P}_{1} \times \mathrm{P}_{4}$ & 1.77 & 1.23 & 1.43 & 2.26 & 1.22 & 1.01 & 1.68 & 1.11 & 1.84 & 1.60 & 1.58 & 1.72 & 1.63 & 0.67 \\
\hline $\mathrm{P}_{1} \times \mathrm{P}_{7}$ & 0.34 & 0.84 & 1.78 & 0.33 & 1.33 & 1.34 & 1.48 & 0.65 & 1.20 & 1.07 & 0.60 & 0.91 & 0.74 & -1.50 \\
\hline $\mathrm{P}_{1} \times \mathrm{P}_{8}$ & 1.30 & 1.41 & 1.97 & 1.54 & 1.62 & 1.50 & 0.54 & 0.66 & 0.64 & 1.27 & 1.89 & 1.46 & 1.34 & -0.52 \\
\hline $\mathrm{P}_{1} \times \mathrm{P}_{9}$ & 0.34 & 1.15 & 1.13 & 1.14 & 0.92 & 1.13 & 1.34 & 0.82 & 1.26 & 1.03 & 1.02 & 1.14 & 1.02 & -0.78 \\
\hline $\mathrm{P}_{1} \times \mathrm{P}_{10}$ & 1.43 & 1.33 & 0.63 & 2.18 & 0.31 & 0.32 & 1.32 & 2.64 & 1.57 & 1.24 & 1.13 & 1.25 & 1.43 & 4.26 \\
\hline $\mathrm{P}_{2} \times \mathrm{P}_{3}$ & 1.63 & 0.67 & 1.20 & 1.88 & 0.91 & 0.99 & 0.57 & -0.40 & 0.52 & 0.93 & 1.24 & 1.19 & 1.03 & -1.35 \\
\hline $\mathrm{P}_{2} \times \mathrm{P}_{4}$ & 1.42 & 0.64 & 1.33 & 0.13 & 1.08 & 0.73 & 0.55 & 0.05 & 1.53 & 1.31 & 1.12 & 1.02 & 0.88 & -1.10 \\
\hline $\mathrm{P}_{2} \times \mathrm{P}_{9}$ & 2.37 & 0.80 & 0.77 & 0.07 & 0.82 & 0.43 & 2.40 & 4.10 & 0.30 & 0.57 & 0.81 & 0.49 & 0.35 & -1.37 \\
\hline $\mathrm{P}_{2} \times \mathrm{P}_{10}$ & 0.60 & 1.01 & 1.15 & 2.15 & 1.28 & 1.11 & 1.32 & 1.88 & 1.81 & 1.86 & 2.28 & 1.63 & 1.56 & 0.86 \\
\hline $\mathrm{P}_{3} \times \mathrm{P}_{4}$ & 1.10 & 1.00 & -0.17 & 0.04 & 1.39 & 1.08 & 0.84 & 1.10 & 0.08 & 0.80 & 1.98 & 0.69 & 0.57 & -0.73 \\
\hline $\mathrm{P}_{3} \times \mathrm{P}_{5}$ & 0.76 & 0.98 & 0.82 & 1.66 & 1.11 & 0.95 & 1.46 & 1.62 & 2.50 & 1.64 & 0.74 & 1.72 & 1.56 & -1.09 \\
\hline $\mathrm{P}_{3} \times \mathrm{P}_{6}$ & 1.26 & 0.82 & 0.17 & 2.00 & 0.65 & 0.58 & 0.21 & 0.96 & 1.48 & 1.19 & 0.99 & 1.58 & 1.45 & -0.44 \\
\hline $\mathrm{P}_{3} \times \mathrm{P}_{7}$ & 0.50 & 1.02 & 1.66 & -0.44 & 0.83 & 1.20 & 0.34 & 0.38 & 1.00 & 1.08 & 1.23 & 0.20 & 0.35 & 1.60 \\
\hline $\mathrm{P}_{3} \times \mathrm{P}_{8}$ & 0.77 & 1.36 & 1.01 & 0.34 & 1.47 & 1.02 & 2.18 & 2.13 & 0.57 & 0.52 & 0.48 & 0.36 & 0.39 & 0.47 \\
\hline $\mathrm{P}_{3} \times \mathrm{P}_{9}$ & 0.44 & 1.03 & 1.58 & 0.30 & 0.93 & 1.33 & 0.10 & 0.05 & 0.63 & 0.56 & 0.66 & 0.68 & 0.52 & -1.47 \\
\hline $\mathrm{P}_{3} \times \mathrm{P}_{10}$ & 0.35 & 0.96 & 0.58 & 1.91 & 0.95 & 0.98 & 0.27 & 0.08 & 0.78 & 0.55 & 0.29 & 1.17 & 1.13 & 0.60 \\
\hline
\end{tabular}


Table 3. Cont'd.

\begin{tabular}{|c|c|c|c|c|c|c|c|c|c|c|c|c|c|c|}
\hline Parents & $\begin{array}{l}\text { Days to } \\
\text { heading }\end{array}$ & $\begin{array}{l}\text { Days to } \\
\text { maturity }\end{array}$ & $\begin{array}{l}\text { Plant } \\
\text { height } \\
(\mathrm{cm})\end{array}$ & $\begin{array}{l}\text { No. of } \\
\text { tillers/ } \\
\text { plant }\end{array}$ & $\begin{array}{c}\text { Flag } \\
\text { leaf } \\
\text { area } \\
\left(\mathrm{cm}^{2}\right) \\
\end{array}$ & $\begin{array}{c}\text { Peduncle } \\
\text { length } \\
(\mathrm{cm})\end{array}$ & $\begin{array}{l}\text { Spike } \\
\text { length } \\
(\mathrm{cm})\end{array}$ & \begin{tabular}{|c} 
No. of \\
spikelets/ \\
spike
\end{tabular} & $\begin{array}{l}\text { No. of } \\
\text { grains/ } \\
\text { spike }\end{array}$ & $\begin{array}{c}\text { Grain } \\
\text { yield/ } \\
\text { spike } \\
\text { (g) }\end{array}$ & $\begin{array}{l}1000- \\
\text { grain } \\
\mathrm{wt} \\
(\mathrm{g})\end{array}$ & $\begin{array}{c}\text { Biological } \\
\text { yield / } \\
\text { plant(g) }\end{array}$ & $\begin{array}{c}\text { Harvest } \\
\text { index }\end{array}$ & $\begin{array}{c}\text { Grain } \\
\text { yield/ } \\
\text { plant } \\
\text { (g) }\end{array}$ \\
\hline 1 & 2 & 3 & 4 & 5 & 6 & 7 & 8 & 9 & 10 & 11 & 12 & 13 & 14 & 15 \\
\hline$\overline{\mathrm{P}_{4} \times \mathrm{P}_{5}}$ & 1.27 & 0.96 & 0.65 & 0.62 & 1.39 & 1.32 & 0.51 & 1.47 & 1.91 & 1.30 & 1.04 & 1.09 & 1.28 & 4.06 \\
\hline $\mathrm{P}_{4} \times \mathrm{P}_{6}$ & 0.93 & 0.81 & 0.85 & 0.71 & 0.83 & 0.96 & 1.84 & 1.23 & 1.88 & 1.76 & 1.83 & 1.27 & 1.45 & 4.60 \\
\hline $\mathrm{P}_{4} \mathrm{xP}_{7}$ & 2.01 & 1.39 & 1.36 & 0.05 & 1.62 & 1.39 & 2.01 & 1.21 & 1.37 & 1.22 & 1.16 & 0.96 & 0.86 & -0.59 \\
\hline $\mathrm{P}_{4} \mathrm{X} \mathrm{P}_{8}$ & 1.16 & 1.01 & 0.88 & 0.02 & 0.96 & 1.42 & 1.71 & 2.41 & 0.52 & 1.12 & 1.72 & 0.27 & 0.68 & 4.28 \\
\hline $\mathrm{P}_{4} \mathrm{xP}_{9}$ & 0.87 & 0.88 & 0.14 & 1.18 & 0.23 & 0.50 & 0.42 & 0.30 & 0.05 & 0.12 & 0.13 & -0.16 & 0.23 & 3.35 \\
\hline $\mathrm{P}_{4} \times \mathrm{P}_{10}$ & 0.61 & 0.91 & 0.11 & 0.72 & 0.83 & 0.52 & 1.04 & 0.89 & -0.37 & 0.70 & 0.02 & 0.89 & 0.89 & 0.82 \\
\hline $\mathrm{P}_{5} \mathrm{xP}_{6}$ & 0.83 & 1.19 & 2.30 & 0.10 & 1.06 & 0.91 & 0.61 & 2.92 & 0.32 & 0.63 & 0.91 & 0.28 & 0.50 & 2.36 \\
\hline $\mathrm{P}_{5} \mathrm{XP}_{7}$ & 1.61 & 0.78 & 1.19 & 0.11 & 0.29 & 0.10 & 0.09 & 0.49 & 1.24 & 0.54 & -0.06 & 0.48 & 0.33 & -1.29 \\
\hline $\mathrm{P}_{5} \mathrm{xP}_{8}$ & 1.70 & 0.79 & 1.18 & 0.19 & 0.77 & 1.16 & 0.84 & 2.01 & 0.53 & 0.47 & 0.41 & 0.28 & 0.09 & -1.78 \\
\hline $\mathrm{P}_{5} \mathrm{xP}_{9}$ & 1.01 & 1.05 & 1.63 & 1.65 & 1.38 & 1.21 & 1.76 & 0.73 & 0.86 & 0.62 & 0.28 & 1.36 & 1.16 & -1.93 \\
\hline $\mathrm{P}_{5} \mathrm{xP}_{10}$ & 0.95 & 1.25 & -0.31 & 2.29 & 0.93 & 0.21 & 0.04 & -1.63 & 1.56 & 1.52 & 1.70 & 1.65 & 1.65 & 2.63 \\
\hline $\mathrm{P}_{6} \mathrm{xP}_{7}$ & 0.84 & 0.86 & 0.80 & 0.03 & 1.10 & 1.50 & 0.28 & 0.14 & 1.26 & 1.06 & 0.94 & 0.27 & 0.54 & 2.82 \\
\hline $\mathrm{P}_{6} \mathrm{X} \mathrm{P}_{8}$ & 1.26 & 1.02 & 0.71 & 0.03 & 1.18 & 1.24 & 0.86 & 0.37 & 1.20 & 0.95 & 0.73 & 0.42 & 0.71 & 3.54 \\
\hline $\mathrm{P}_{6} \times \mathrm{P}_{9}$ & 0.96 & 0.95 & 0.94 & 0.11 & 0.64 & 0.96 & 1.16 & 1.08 & 0.33 & 0.79 & 1.18 & 0.86 & 0.74 & -0.87 \\
\hline $\mathrm{P}_{6} \mathrm{XP}_{10}$ & 0.53 & 1.24 & 0.90 & 2.67 & 1.28 & 1.12 & 1.38 & 1.25 & 0.54 & 0.98 & 1.39 & 1.61 & 1.49 & -0.45 \\
\hline $\mathrm{P}_{7} \mathrm{X} \mathrm{P}_{8}$ & 1.01 & 1.15 & 1.03 & 0.10 & 0.43 & 1.47 & 0.75 & 1.53 & 0.29 & 0.74 & 1.17 & 0.31 & 0.44 & 1.54 \\
\hline $\mathrm{P}_{7} \mathrm{xP}_{9}$ & 0.43 & 0.73 & 0.39 & 0.06 & 0.09 & 0.68 & 0.63 & 1.53 & 0.52 & 0.31 & 0.53 & 0.38 & 0.31 & -0.52 \\
\hline $\mathrm{P}_{7} \mathrm{XP}_{19}$ & 0.62 & 1.24 & 0.10 & 2.04 & 0.32 & 0.65 & 0.51 & 0.22 & 0.83 & 0.52 & 0.19 & 1.23 & 1.05 & -1.55 \\
\hline $\mathrm{P}_{8} \times \mathrm{P}_{9}$ & 0.70 & 0.79 & 1.47 & 0.35 & 1.45 & 1.35 & 2.06 & 0.59 & 1.92 & 1.18 & 0.40 & 0.98 & 1.20 & 4.20 \\
\hline $\mathrm{P}_{8} \times \mathrm{P}_{10}$ & 0.35 & 1.06 & 1.26 & 1.44 & 1.54 & 1.29 & 1.91 & 0.95 & 1.44 & 0.82 & 0.16 & 1.10 & 1.27 & 3.85 \\
\hline $\mathrm{P} 9 \mathrm{xP}_{10}$ & 0.61 & 1.13 & 0.06 & 1.34 & 0.11 & 0.43 & 0.42 & 0.84 & 0.83 & 1.05 & 1.31 & 0.91 & 1.22 & 5.24 \\
\hline D-value & 0.047 & 0.097 & 0.099 & 0.163 & 0.360 & 0.276 & 0.112 & 0.064 & 0.153 & 0.292 & 0.159 & 0.370 & 0.043 & 0.396 \\
\hline
\end{tabular}


Table 4. Heat susceptibility indices of $F_{2}$ crosses for various characters in bread wheat.

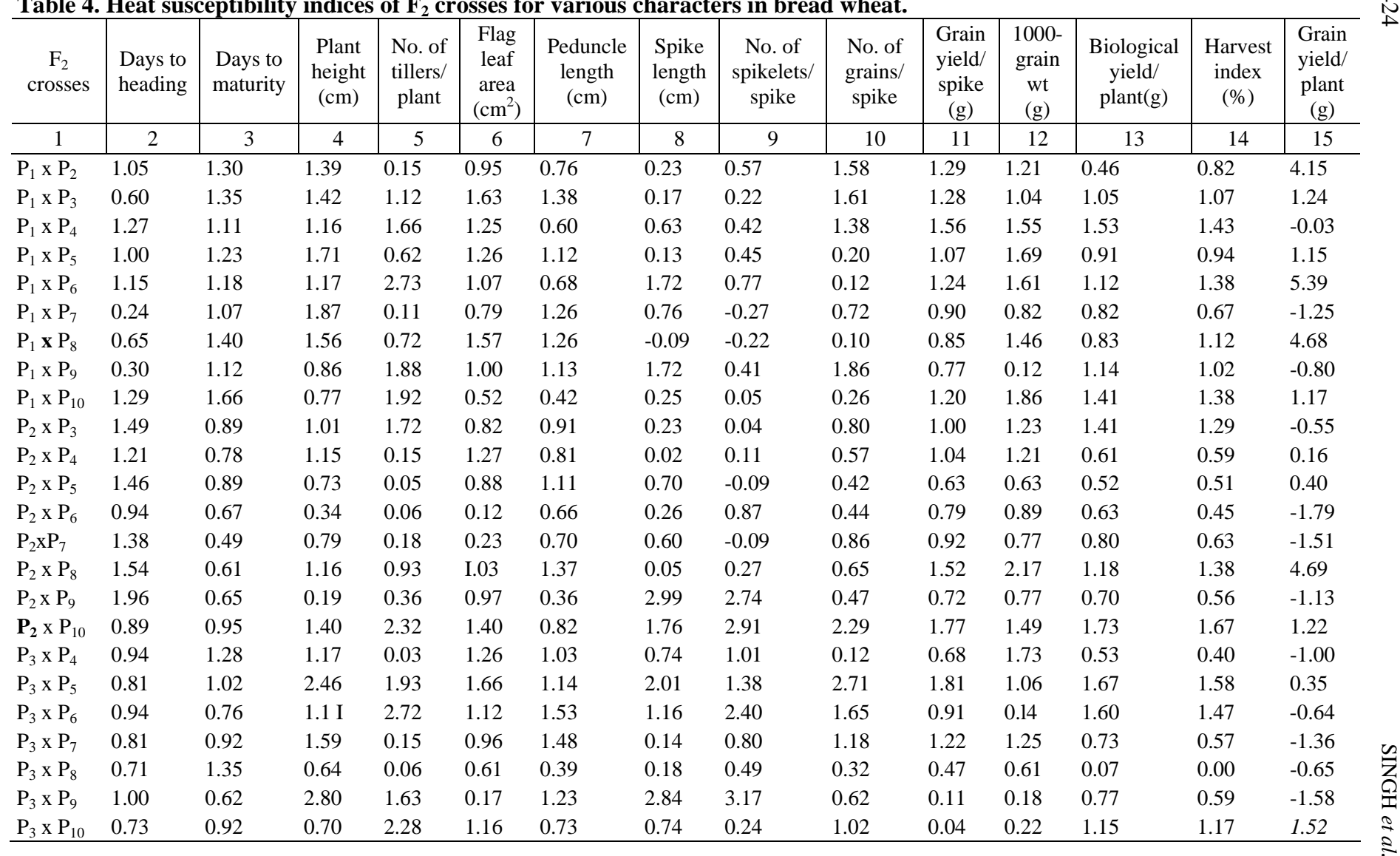


Table 4. Cont'd.

\begin{tabular}{|c|c|c|c|c|c|c|c|c|c|c|c|c|c|c|}
\hline $\begin{array}{c}\mathrm{F}_{2} \\
\text { crosses }\end{array}$ & $\begin{array}{l}\text { Days to } \\
\text { heading }\end{array}$ & $\begin{array}{l}\text { Days to } \\
\text { maturity }\end{array}$ & \begin{tabular}{|} 
Plant \\
height \\
$(\mathrm{cm})$
\end{tabular} & $\begin{array}{c}\text { No. of } \\
\text { tillers/ } \\
\text { plant }\end{array}$ & $\begin{array}{l}\text { Flag } \\
\text { leaf } \\
\text { area } \\
\left(\mathrm{cm}^{2}\right) \\
\end{array}$ & $\begin{array}{c}\text { Peduncle } \\
\text { length } \\
(\mathrm{cm})\end{array}$ & $\begin{array}{l}\text { Spike } \\
\text { length } \\
(\mathrm{cm})\end{array}$ & $\begin{array}{c}\text { No. of } \\
\text { spikelets/ } \\
\text { spike }\end{array}$ & $\begin{array}{l}\text { No. of } \\
\text { grains/ } \\
\text { spike }\end{array}$ & $\begin{array}{l}\text { Grain } \\
\text { yield/ } \\
\text { spike } \\
\text { (g) }\end{array}$ & $\begin{array}{l}\text { 1000- } \\
\text { grain } \\
\text { wt } \\
\text { (g) }\end{array}$ & $\begin{array}{c}\text { Biological } \\
\text { yield/ } \\
\text { plant(g) }\end{array}$ & $\begin{array}{c}\text { Harvest } \\
\text { index } \\
(\%)\end{array}$ & $\begin{array}{c}\text { Grain } \\
\text { yield/ } \\
\text { plant } \\
\text { (g) }\end{array}$ \\
\hline 1 & 2 & 3 & 4 & 5 & 6 & 7 & 8 & 9 & 10 & 11 & 12 & 13 & 14 & 15 \\
\hline$\overline{\mathrm{P}_{4} \times \mathrm{P}_{5}}$ & 1.01 & 0.75 & 0.46 & 1.87 & 1.07 & 0.77 & 0.72 & 2.06 & 1.23 & 1.23 & 1.22 & 1.41 & 1.30 & -0.53 \\
\hline $\mathrm{P}_{4} \times \mathrm{P}_{6}$ & 1.11 & 0.94 & 0.37 & 0.37 & 1.15 & 1.03 & 3.24 & 2.32 & 1.52 & 1.52 & 1.47 & 1.12 & 1.20 & 2.38 \\
\hline $\mathrm{P}_{4} \mathrm{xP}_{7}$ & 1.61 & 1.18 & 0.75 & 0.05 & 1.57 & 1.15 & 1.80 & 0.11 & 1.83 & 1.63 & 1.56 & 1.22 & 1.12 & -0.50 \\
\hline $\mathrm{P}_{4} \times \mathrm{P}_{8}$ & 1.09 & 0.84 & 0.89 & 0.77 & 1.15 & 1.57 & 1.72 & 1.10 & 0.46 & 0.86 & 1.22 & 0.30 & 0.67 & 4.01 \\
\hline $\mathrm{P}_{4} \mathrm{xP}_{9}$ & 1.07 & 0.56 & 0.86 & 0.27 & 0.98 & 0.96 & 2.42 & 2.77 & 0.47 & 0.06 & 0.15 & 0.40 & 0.35 & -0.16 \\
\hline $\mathrm{P}_{4} \times \mathrm{P}_{10}$ & 0.95 & 0.90 & 0.43 & 0.09 & 0.44 & 0.51 & 0.03 & -0.16 & 1.63 & 0.97 & 0.25 & 0.81 & 0.80 & 0.45 \\
\hline $\mathrm{P}_{5} \mathrm{xP}_{6}$ & 1.27 & 1.14 & 1.03 & 0.06 & 0.75 & 0.67 & 0.17 & 0.76 & 0.02 & 0.38 & 0.86 & 0.15 & -0.02 & -1.70 \\
\hline $\mathrm{P}_{5} \mathrm{xP}_{7}$ & 1.56 & 0.88 & 0.74 & -0.78 & 0.46 & 0.04 & 0.15 & 1.24 & 0.79 & 0.30 & 0.04 & 0.04 & 0.25 & 1.92 \\
\hline $\mathrm{P}_{5} \mathrm{xP}_{8}$ & 1.63 & 0.81 & 1.03 & 0.22 & 0.88 & 1.27 & 1.09 & 2.68 & 1.00 & 0.68 & 0.16 & 0.59 & 0.86 & 3.55 \\
\hline $\mathrm{P}_{5} \mathrm{xP}_{9}$ & 1.16 & 1.12 & 0.54 & 1.27 & 1.48 & 0.64 & 1.36 & 0.03 & 0.75 & 1.00 & 1.18 & 1.12 & 1.10 & 0.76 \\
\hline $\mathrm{P}_{5} \mathrm{xP}_{10}$ & 0.84 & 0.97 & 0.12 & 1.86 & 0.29 & 0.84 & 0.19 & 2.06 & 1.83 & 1.83 & 1.96 & 1.56 & 1.71 & 5.85 \\
\hline $\mathrm{P}_{6} \mathrm{xP}_{7}$ & 1.01 & 0.99 & 0.54 & 0.08 & 0.79 & 1.02 & 0.22 & 0.91 & 0.86 & 0.90 & 1.03 & 0.30 & 0.27 & -0.06 \\
\hline $\mathrm{P}_{6} \times \mathrm{P}_{8}$ & 0.49 & 0.94 & 0.39 & 0.25 & 1.11 & 1.42 & 1.58 & 0.52 & 1.08 & 0.72 & 0.22 & 0.72 & 0.61 & -0.94 \\
\hline $\mathrm{P}_{6} \times \mathrm{P}_{9}$ & 0.96 & 1.07 & 0.43 & 0.27 & 0.91 & 1.04 & 2.09 & 1.60 & 0.68 & 0.83 & 1.00 & 0.77 & 0.78 & 0.78 \\
\hline $\mathrm{P}_{6} \mathrm{XP}_{10}$ & 0.78 & 1.00 & 1.17 & 2.50 & 1.50 & 1.23 & 1.47 & 0.23 & 1.11 & 1.10 & 1.11 & 1.51 & 1.40 & -0.24 \\
\hline $\mathrm{P}_{7} \mathrm{X}_{\mathrm{P} 8}$ & 0.87 & 1.07 & 0.84 & 0.08 & 0.74 & 1.22 & 1.39 & 2.82 & 0.44 & 0.89 & 1.17 & 0.64 & 0.60 & 0.03 \\
\hline $\mathrm{P}_{7} \mathrm{xP}_{9}$ & 0.55 & 0.98 & 0.63 & 0.89 & 0.53 & 1.48 & 0.06 & 1.98 & 0.75 & 0.73 & 0.83 & 1.10 & 0.95 & -1.19 \\
\hline $\mathrm{P}_{7} \mathrm{XP}_{10}$ & 0.79 & 0.99 & 1.20 & 0.62 & 0.04 & 1.14 & 0.62 & 0.06 & 0.55 & 0.58 & 0.50 & 0.74 & 0.74 & 0.38 \\
\hline $\mathrm{P}_{8} \times \mathrm{P}_{9}$ & 0.95 & 0.82 & 1.06 & 0.13 & 1.23 & 1.02 & 1.26 & 0.93 & 1.74 & 1.12 & 0.37 & 0.37 & 0.75 & 4.30 \\
\hline $\mathrm{P}_{8} \times \mathrm{P}_{10}$ & 0.06 & 1.41 & 1.61 & 1.59 & 1.29 & 1.24 & 0.20 & 0.20 & 1.23 & 0.89 & 0.44 & 1.05 & 1.25 & 4.09 \\
\hline $\mathrm{P}_{9} \mathrm{XP}_{10}$ & 0.73 & 1.42 & 0.06 & 1.08 & 0.71 & 0.93 & 0.21 & 0.30 & 1.21 & 1.31 & 1.12 & 0.74 & 1.02 & 4.26 \\
\hline D-value & 0.067 & 0.098 & 0.111 & 0.161 & 0.329 & 0.289 & 0.087 & 0.054 & 0.141 & 0.285 & 0.176 & 0.386 & 0.045 & 0.413 \\
\hline
\end{tabular}


Most of the traits were adversely affected under late sown conditions. It was, therefore, essential to develop a scale for sorting parents and crosses for their relative tolerance to high temperature. In the present study that based on HSI (i.e. below I), the parental genotype; HD 2851, PBW 520, and HS 448 and the crosses HS $448 \times$ PBW 520, Up $2614 \times \mathrm{K}$ 209, and PBW $520 \times$ HD 2851 were least affected under late sown conditions $\left(\mathrm{F}_{2}\right)$. Parent HD 2851 and the cross PBW 520 $\times$ HD 2851 also exhibited good GCA and SCA effects for grain yield per plant, respectively, in late sown environment $\left(\mathrm{F}_{2}\right)$. These parents and crosses should be further exploited for improvement of grain yield under late sown conditions. The HSI could be taken as important criteria for breeding wheat genotypes suitable for late sown conditions.

\section{References}

Blum, A., C. Y. Sullivan and H. T. Naguyen. 1997. The effect of plant size of wheat in responses to drought stress II. Water deficit, heat and ABA. Aust. J. Plant Physiol. 24: 43-48.

FAO 2010. htt9://www.fao.org/docrop/0 11 /ai482e/ai482e03 .htm

Fisher, R. A. and R. Maurer. 1978. Drought resistance in spring wheat cultivars I. Grain yield responses. Aust. J. Agric. Res., 29: 897-912.

Nagarajan, S. and J. Rane. 2002. Physiological traits associated with yield performance of spring wheat (Triticum aestivum L.) under late sown condition. Indian .J. Agric. Sci. 72: 135-140.

Prasad. R. and S. Nagarajan. 2004. Rice wheat cropping system Food Security and Sustainability. Cur. Sci. 87: 1334-1335.

Rashid, M. H., S. C. Samanta, P. Biswas M. A. Mannan and A. K. M. M. Zaman. 2004. Performance of thirty wheat genotypes under late sowing conditions in southern region of Bangladesh. Asian J. Plant Sci, 3: 286-289.

Sharma, R. C. 1993. Selection for biomass yield in wheat. Euphytica 70: 35-42.

Ved Praksh. 2007. Screening of wheat (Triticum aestivum L.) genotypes under limited moisture and heat stress environments. Indian J. Genet. 67: 31- 33.

Vivek Raj and A. K. Bhardwaj. 2004. Physiological parameters of wheat cultivars as affected by time of sowing in lndo-gangetic Plain of U.P. and Uttaranchal. $J$. Farming Systems Research and Development 10: 158-159. 